\title{
Pre-discharge defibrillation testing: Is it still justified?
}

\author{
Maciej Kempa, Tomasz Królak, Łukasz Drelich, Szymon Budrejko, \\ Ludmiła Daniłowicz, Ewa Lewicka, Dariusz Kozłowski, Grzegorz Raczak
}

Department of Cardiology and Electrotherapy, Medical University of Gdansk, Poland

\begin{abstract}
Background: An implantable cardioverter-defibrillator (ICD) is routinely used to prevent sudden cardiac death. Since the introduction of that device into clinical practice, a defibrillation test (the so-called pre-discharge test [PDT]) has been an inseparable part of the ICD implantation procedure. Recently, the usefulness of PDT has been called into question.

Methods: The aim of this research was to analyze ICD tests performed within two time periods: in years 1995-2001 (period I) and 2007-2010 (period II), in order to compare the results of tests and solutions to all the problems with ICD systems revealed by means of PDT.

Results: During period I, 193 tests were performed, among which the ICD system malfunction was observed in 16 cases. Those included: sensing issues, specifically $R$-wave undersensing during ventricular fibrillation (VF) (7 patients) and T-wave oversensing (4 patients), as well as high defibrillation threshold (DFT) (2 patients) and ICD-pacemaker interaction (3 patients). During period II, among 561 tests, system malfunction was observed in 15 cases. In 1 patient it was VF undersensing, and in the remaining 14 it was high DFT. All the above problems were solved by means of appropriate ICD reprogramming, repositioning of the endocardial defibrillation lead or implantation of an additional subcutaneous defibrillation lead.

Conclusions: Contemporary ICD technical solutions, compared to older systems, in most cases allow to avoid sensing problems. The key rationale behind ICD testing is the ability to confirm the efficacy of high-voltage therapy. Despite the increasing maximal defibrillation output of devices, and all possible adjustments to the characteristics of the impulse, there is still a group of patients that require additional procedures to ensure the appropriate defibrillation efficacy. (Cardiol J 2016; 23, 5: 532-538)
\end{abstract}

Key words: pre-discharge test, PDT, implantable cardioverter-defibrillator, ICD

\section{Introduction}

An implantable cardioverter-defibrillator (ICD) is commonly used to prevent sudden cardiac death (SCD) due to ventricular arrhythmias. Since the introduction of that device into clinical practice, a defibrillation test (so-called pre-discharge test [PDT]) has been an inseparable part of the ICD implantation procedure. PDT used to be performed during ICD implantation procedure or immediately after the procedure, but increasingly often it takes place several days after implantation. It is based on the induction of ventricular fibrillation (VF), which is then detected and terminated by an ICD shock, which depends on the protocol of the test and the ICD model. The main aim of the test is to

Address for correspondence: Maciej Kempa, MD, PhD, Department of Cardiology and Electrotherapy, Medical University of Gdansk, ul. Dębinki 7, 80-211 Gdańsk, Poland, e-mail: kempa@gumed.edu.pl

Received: 27.06.2016

Accepted: 29.06.2016 
Table 1. Implantable cardioverter-defibrillator (ICD) implantation procedures and ICD tests in both analyzed periods.

\begin{tabular}{lccc}
\hline & $\begin{array}{c}\text { Period I } \\
\text { (years 1995-2001) }\end{array}$ & $\begin{array}{c}\text { Period II } \\
\text { (years 2007-2010) }\end{array}$ & P \\
\hline Total number of ICD implantation procedures & 228 & 610 & - \\
Total number of ICD tests & 228 & 561 & - \\
Number of tests included in the analysis & 193 & 556 & - \\
Number of tests without ventricular fibrillation induction & $0(0 \%)$ & $5(0.9 \%)$ & NS \\
Number of tests that revealed system malfunction & $16(8.3 \%)$ : group A & $15(2.7 \%):$ group B $<0.001$ \\
\hline
\end{tabular}

confirm the appropriate arrhythmia detection, as well as the efficacy of the defibrillation impulse (the energy of which usually equals the maximum ICD output less $10 \mathrm{~J}$ ). PDT also proves the integrity of the ICD-lead(s) system and its connections, allows to assess the sensing/detection and pacing settings, and to develop the final program of the device. Sometimes, the PDT is used to establish the lowest energy that terminates VF, the so-called defibrillation threshold (DFT).

In recent years, some authors have called into question the usefulness of routine PDT. The key argument is that most information resulting from PDT can be acquired by other means. Moreover, execution of PDT does not influence mortality of ICD recipients, which in the light of the risk of PDT itself puts the usefulness of that procedure in question $[1,2]$. On the other hand, one might argue that in some patients, the result of PDT brings useful information and influences further clinical approach. Moreover, in recent years, we have observed considerable progress in cardiac electrotherapy and there is clearly a need to compare former PDT results with those of today in terms of their clinical significance.

The aim of the study was to compare the results of ICD testing and the following strategy to solve all the clinical problems revealed during the tests. We investigated the change in PDT results over 15 years of ICD use in our department and in the management strategy of ICD-recipients. For that purpose, we retrospectively analyzed the data of patients that have undergone ICD implantation in our department in two time periods: in years 1995-2001 and 2007-2010 (Table 1).

\section{Methods}

Group A was selected from 228 consecutive patients that underwent an ICD implantation procedure in years 1995-2001 (period I). Thirty-five patients were excluded from that group due to lack of Holter memory in the implanted device, which would allow to record intracardiac electrograms (Phylax 03, Biotronik), or due to incomplete medical history. As a result, 193 patients (52 female, 141 male) at the age of $18-80$ (mean $55.8 \pm 15.3$ ), who had a PDT performed after the implantation procedure, turned out to be eligible for further analysis. In that group, the system malfunction was discovered during the PDT in 16 patients (group A). That group consisted of 5 women and 11 men who were $30-80$ years of age (mean $55 \pm 14.4$ ). The primary underlying condition was coronary artery disease (CAD) in 13 patients, dilative cardiomyopathy (DCM) in 2 patients, and long QT syndrome in 1 patient. Three patients had an additional pacemaker implanted (1 - AAI, 1 - DDD, 1 - VVI). Left ventricular ejection fraction (LVEF) was 20-70\% (mean 42.1 \pm 16.9 ). All patients had undergone sudden cardiac arrest and were qualified for an ICD in secondary prevention of SCD. All the devices but one were single-chamber, manufactured by Biotronik: Phylax 06 - 6 patients, Phylax $\mathrm{XM}-4$ patients, Mycrophylax -5 patients. One patient received a dual-chamber ICD - Phylax AV, also manufactured by Biotronik.

Group B was selected from 610 consecutive patients that underwent ICD implantation procedure in years 2007-2010 (period II). In that group, a PDT was conducted in 561 patients (131 female, 430 male), at the age of $18-92$ (mean $61.6 \pm 13.1$ ). PDT was not performed in 49 patients due to typical contraindications (persistent atrial fibrillation or artificial valve prosthesis without appropriate anticoagulation, cardiac chamber thrombus, hemodynamic instability, significant aortic stenosis, prior stroke, symptomatic CAD, CAD not eligible for revascularization). In 5 cases, VF could not be induced during PDT, despite repeated attempts and various induction protocols. The system malfunction was revealed during PDT in 15 patients and 
that group constituted a subject for further analysis (group B). It consisted of 2 women and 13 men, at the age of 32-68 (mean $51.2 \pm 10.3$ ). The underlying condition was CAD in 7 patients, DCM in 6 patients, and laminopathy in 1 patient. LVEF range was $15-65 \%$ (mean $28.3 \pm 13.5$ ). In 6 patients, the ICD had been implanted in the secondary prevention, and in 9 patients, in the primary prevention of SCD. The devices were as follows: single-chamber in 5 patients, dual-chamber in 5 , and bi-ventricular in 5 patients. The devices were manufactured by Biotronik (Lumos DR - 1, Lumax $340 \mathrm{VR}-1$, Lumax $340 \mathrm{DR}-1$ ), Medtronic (Insync Sentry - 3, Insync Maximo - 1, Maximo VR - 4, Maximo DR - 1, Maximo II DR - 1, Maximo II CRT - 1) and Boston Scientific (Teligen 100 DR - 1).

Pre-discharge tests were performed in an electrophysiology laboratory, 1-30 days after the ICD implantation procedure. Following the thorough sensing and pacing parameters control, VF was induced under short-term general anesthesia, instituted by an anesthesiologist. Shock-on-T or burst pacing were used as VF induction methods. The PDT protocol was different in both of the analyzed periods. In years 1995-2001, the test consisted of repeated VF induction and defibrillation with an adjusted energy, aimed at determining the DFT (the lowest energy sufficient to terminate the arrhythmia and restore the sinus rhythm). The first energy value tested was $13 \mathrm{~J}$. Subsequently, $8 \mathrm{~J}, 4.5 \mathrm{~J}$, and $3.1 \mathrm{~J}$ values were tested. If the first $13 \mathrm{~J}$ value, shock was inefficient, the energy value of $21 \mathrm{~J}$ was tested, and if that in turn was effective, the $18 \mathrm{~J}$ shock followed. The PDT result was satisfactory if $\mathrm{VF}$ was terminated twice with a shock of no more than $21 \mathrm{~J}$. In years $2007-2010$, a single defibrillation efficacy test was used, without determination of the DFT. For the time of the test, the ICD was programmed to deliver the first shock of maximum energy of a particular device of less $10 \mathrm{~J}$ $(\max -10 \mathrm{~J})$ or less $15 \mathrm{~J}(\max -15 \mathrm{~J})$. The second shock was the maximum energy of the device in the former case, and the maximum energy less $10 \mathrm{~J}$ in the latter case. All the following shocks were of the maximum available output. The test was deemed satisfactory if VF was terminated by the shock max $-10 \mathrm{~J}$ or max $-15 \mathrm{~J}$. During both analyzed periods, if the test was unsatisfactory (period I: DFT $>21$ J, period II: DFT $>$ max $-10 \mathrm{~J}$ ) the test was repeated with reversed shock polarity. In group A, 5 out of 16 patients had a dual-coil lead, while in group B it was 1 out of 15 patients. The shock configuration was initially set as default setting of every ICD. In dual-coil systems, it was always distal shocking electrode (HVB) to the proximal electrode (SVC) and to the pulse generator case (can). If the measures described above were insufficient to achieve the pre-set defibrillation safety margin, the patient was qualified for the defibrillation lead repositioning (period I) or the implantation of a subcutaneous defibrillation lead (period II).

Our study had completely retrospective character based on medical history analysis no personal data of the patients was used and due to this the bioethical committee opinion was not needed.

\section{Statistical analysis}

All statistical analyses were performed with the use of Statistica 10 PL, StatSoft. Descriptive statistics used numbers and percentages, mean value and standard deviation, and the maximum and minimum values. Normal distribution of continuous variables was assessed with the Shapiro-Wilk W test. Variance homogeneity was tested with the Levene's test. Variables with normal distribution and homogenous variances were compared using the Student's t-test for unrelated variables. If variances were not homogenous, the CochraneCox test was used. If normal distribution could not be confirmed, variables were compared with the Mann-Whitney U test. In all the analyses, the border confidence value for rejection of the null hypothesis was set as $\alpha=0.05$. The calculated p-value for each test, if lower than 0.05 , was labeled adequately as: $\mathrm{p} \leq 0.05, \mathrm{p} \leq 0.01$, and $\mathrm{p} \leq 0.001$.

\section{Results}

During period I, the system malfunction was observed in 16 cases among 193 ICD tests (group A). The problems were: detection disorders $\mathrm{R}$ wave undersensing during $\mathrm{VF}$ (7 patients), T-wave oversensing (4 patients), high DFT (2 patients) and ICD-pacemaker interactions - oversensing of atrial pacing impulse of the AAI pacemaker in the ventricular channel of the ICD (1 patient), oversensing of ventricular pacing impulse of the DDD pacemaker in the ventricular channel of the ICD ( 1 patient) and undersensing of VF due to uninhibited ventricular pacing of the VVI pacemaker during VF (1 patient). During period II, system malfunction was observed in 15 cases among 561 ICD tests. In 1 case, it was undersensing of VF, and in the remaining 14 cases - high DFT (group B). These data are presented in Table 2. Numbers of patients in both groups and tests and system malfunctions are summarized in Figure 1. 
Table 2. Detailed data of patients in groups $A$ and $B$, and system malfunctions during implantable cardioverter-defibrillator (ICD) tests in both groups.

\begin{tabular}{lccc}
\hline & Group A & Group B & P \\
\hline Number of patients & 16 & 15 & $<0.001$ \\
Number of M/F (percentage of M/F) & $11 / 5(69 \% / 31 \%)$ & $13 / 2(87 \% / 13 \%)$ & $\mathrm{NS}$ \\
Age & $30-80($ mean $55 \pm 14.4)$ & $32-68($ mean $51.2 \pm 10.3)$ & $\mathrm{NS}$ \\
Left ventricular ejection fraction & $20-70 \%$ (mean $42.1 \pm 16.9)$ & $15-65 \%($ mean $28.3 \pm 13.5)$ & $<0.001$ \\
Undersensing of VF & $7 / 193=3.6 \%$ & $1 / 561=0.2 \%$ & $<0.001$ \\
T-wave oversensing & $4 / 193=2.1 \%$ & $0 / 561=0 \%$ & $<0.001$ \\
PM-ICD interactions & $3 / 193=1.6 \%$ & $0 / 561=0 \%$ & $\mathrm{NS}$ \\
High defibrillation threshold & $2 / 193=1 \%$ & $15 / 561=2.7 \%$ & \\
\hline
\end{tabular}

$\mathrm{F}$ - female; $\mathrm{M}$ - male; $\mathrm{PM}$ - pacemaker; VF — ventricular fibrillation

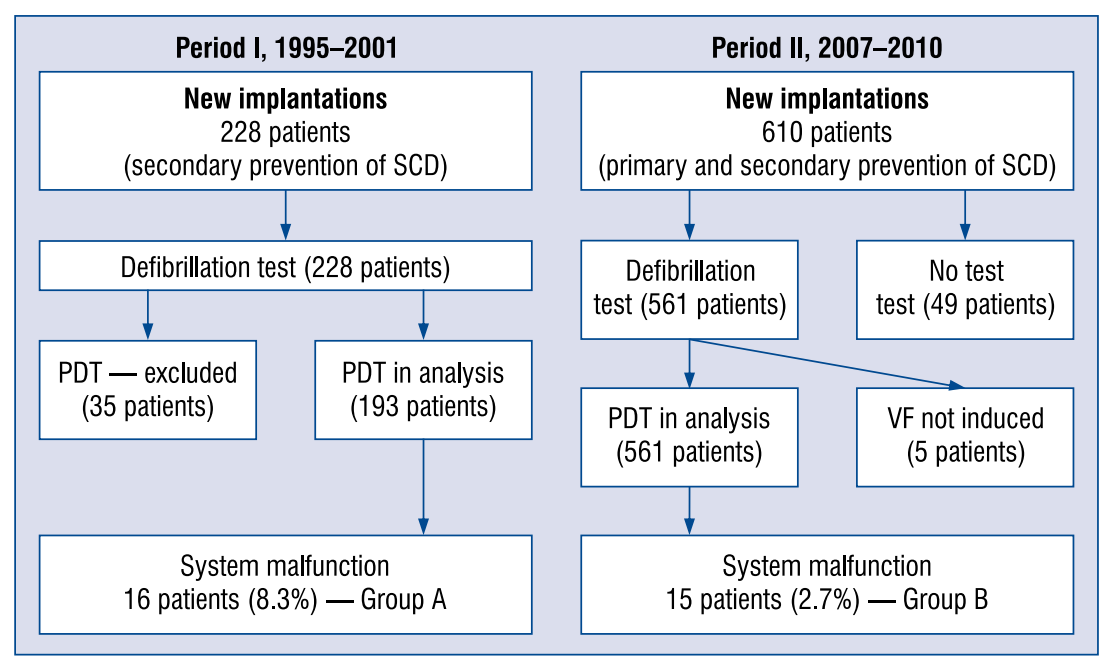

Figure 1. Diagram summarizing numbers of patients, tests and system malfunctions in both study groups; PDT — pre-discharge test; SCD — sudden cardiac death; VF — ventricular fibrillation.

\section{Troubleshooting of problems revealed during $\mathrm{PDT}$}

In case of R-wave undersensing in group A, the following program changes were introduced: an increase of the ICD ventricular sensitivity (6 patients), reduction of the number of intervals for VF detection ("X of Y criterion" - 3 patients), turning off the confirmation of detection before shock ("confirmation OFF" -2 patients), reprogramming of the $\mathrm{VF}$ detection window to the lower value (2 patients), and inversion of the ventricular intracardiac signal (3 patients). In all patients with T-wave oversensing, the filtration settings of intracardiac electrogram were changed. In order to exclude the ICD-pacemaker interactions, the pacing impulse configuration was changed to bipolar, with the lowest possible output value, and with the maximum sensing setting of the pacemaker. In case of high DFT, the polarity of the defibrillation impulse was reversed. One patient required double repositioning of the defibrillation lead.

The repeated ICD test after having introduced the above measures, proved their efficacy - no detection problems were observed, and successful defibrillation was achieved with the energy value of $\leq 21 \mathrm{~J}$.

In group $\mathrm{B}$, in case of $\mathrm{VF}$ undersensing, the device sensitivity was increased (1 patient). In case of high DFT, the reversed polarity impulse was tested. It was satisfactory in 4 patients, and the remaining 10 patients required the implantation of an additional subcutaneous defibrillation lead.

\section{Discussion}

At the end of the last century, some authors already advised abandoning the routine of ICD testing $[3,4]$. The usefulness of PDT is questioned also 
contemporarily [5-7]. According to some experts, most of the essential information can be acquired during normal follow-up of the device. That includes confirmation of appropriate sensing with the sensing test, or the proof of correct ICD-lead connection by the means of electrical impedance measurements. There is also some doubt about the efficacy of a defibrillation test, which is the most crucial part of PDT. According to some experts, successful defibrillation has low repeatability, as it is influenced both by numerous clinical (drugs, hypoxia, anemia, acidosis, electrolyte imbalance, increase of the left ventricular wall thickness) and physical conditions (concerning the characteristics of a defibrillation impulse itself). Moreover, the controversy is augmented by the variety of PDT protocols and lack of guidelines for proper interpretation of test results. The question still remains unanswered, whether the arrhythmia induced during PDT is clinically relevant, and whether it is reasonable to test responses to VF, as most real-life episodes recorded by ICDs are ventricular tachycardias. An important argument against PDT is the risk of general anesthesia and VF induction. Finally, it should be emphasized that data from clinical trials did not show a significant mortality reduction that could be expected for PDT and the additional confirmation of ICD system integrity and function $[8,9]$. The results of the SIMPLE trial which did not show any benefit of performing a PDT, seem to be important in that matter [10]. Importantly, retrospective observations, however, are still of interest for clinicians, as they show results based on real-life subsets of all consecutive patients [11]. Thus, in our opinion, despite all doubts, ICD tests should be routinely used, as in some patients, the PDT result significantly influences the further clinical approach. This includes the modification of sensing parameters in case of sensing issues during PDT, as well as the change of shock characteristics, the implantation of a subcutaneous defibrillation lead, or device replacement for a higher-energy model in case of high DFT.

The aim of this study was to assess, whether the results of PDT during 15 years of ICD use in our department justify the decision to abandon the routine of ICD testing. Our data show that current ICDs have sensing and detection algorithms superior to older ones. Appropriate programmable filters, in most cases pre-determined with no further detailed modification options, as well as digital processing of the intracardiac electrogram, almost entirely eliminate sensing and detection issues. Such system malfunction was observed in
$11(5.7 \%)$ cases during period I and merely in $1(0.2 \%)$ case during period II $(\mathrm{p}<0.001)$. It was mainly VF undersensing in older ICD models in 7 (3.6\%) cases. That result is comparable to other reports from that era [3, 12, 13]. During period II, undersensing was observed only in 1 case. Also, the T-wave oversensing (4 cases in years 1995-2001) was almost entirely eliminated (no such case in years 2007-2010) ( $\mathrm{p}<0.001)$. The introduction of dual-chamber and bi-ventricular ICDs eliminated the problem of ICD-pacemaker interactions in patients that require both permanent pacing and an ICD. In the cases described in the article, AAI and DDD pacemakers were left due to the need for atrial pacing and the lack of that possibility in the ICD systems that were available at that time in our country. The VVI pacemaker was left to prevent ICD battery use for ventricular pacing, especially if the PM system was implanted long before the ICD, and the lack of technical ability to extract leads at that time. Obviously,, in the modern era, this notion is never used again, but to our knowledge, in the pioneering ICD era, the co-existence of ICD and pacemakers was not that rare.

What is of great significance, the high DFT still remains an issue. In our cohort of patients with modern ICD systems, during period II, it was observed even in a higher percentage, although this difference did not reach statistical significance. That result is close or slightly lower than reported by other authors. Osswald et al. [14] observed high defibrillation threshold in $6.3 \%$ of patients. Verma et al. [15] defined high defibrillation threshold as an unsuccessful $25 \mathrm{~J}$ shock, observed that phenomenon in $5.1 \%$ of patients, and implanted subcutaneous leads in all such patients with final success. Brigniole et al. [6] did not achieve a $10 \mathrm{~J}$ safety margin in $7 \%$ of patients in their group, and in $3.6 \%$ of patients even the shock with maximal output energy was not efficient. It may result from the fact that in our report, the high DFT was defined as DFT of more than $21 \mathrm{~J}$ (period I) or more than the maximum energy output of the device less $10 \mathrm{~J}$ (period II). As various ICD models were used, in some patients in group B, high DFT means the DFT value of $>25 \mathrm{~J}$, and in some of $>30 \mathrm{~J}$. In the literature, high DFT was defined as the value of $>18 \mathrm{~J}$, especially in the era when the maximum output was not higher than $30 \mathrm{~J}$. Because of that, the incidence of high DFT in particular publications may vary. In the most recent SIMPLE study cohort, in the group that was subject to defibrillation testing, the high DFT (above $21 \mathrm{~J}$ ) was observed in 99 of 1,218 patients (which is $8.1 \%$ ). In our study, 
the greater incidence of high DFT in group B may have several explanations: in group A, 5 out of 16 patients had a dual-coil lead, while in group B, it was 1 out of 15 patients. The LVEF value tended to be lower in group B (although it was not statistically significant). During period II, more patients had biventricular devices. And, in older times, patients were more often implanted in secondary prevention (with primary VF in otherwise organically healthy hearts), while contemporarily the tendency is to implant ICDs in primary prevention of SCD. All of the abovementioned factors may have led to the divergent percentage of high DFT. It is important to underline that during both periods of time taken into our analysis, the scheme of defibrillation testing was different (multiple inductions in period I, as limited induction as possible in period II). It is a result of different routine of defibrillation testing in those periods, which changed in time with emerging scientific evidence. It cannot be excluded that different protocols might have led to divergent results, and that is one of possible limitations of our analysis, however major problems revealed during the test during the period I were associated with sensing and pacemaker-ICD interactions, which were not dependent on the actual number of inductions and subsequent defibrillation setting.

The problem of high DFT justifies the use of PDT. It would be inappropriate to avoid ensuring patients that the device intended to save their lives works properly right away from the implantation procedure. Can one ignore the unsuccessful test defibrillation performed in clinically controlled conditions, in a stable patient, that has been carefully prepared for the test? Can one expect the efficacy of such a device during the follow-up, in case of spontaneous arrhythmia, that is likely to occur during circulatory decompensation or ischemia, which further decrease the likelihood of the arrhythmia termination? It seems that the ICD system that acutely fails to perform its intended role requires modification or replacement. That condition can only be confirmed with PDT. Recently, even more doubt about the value of defibrillation testing has been raised by the results of the SIMPLE trial, in which the no-testing turned out to be non-inferior to the testing strategy in terms of a combined endpoint of arrhythmic death or unsuccessful first shock [10]. In our opinion however, and to what we understand from the results of that trial, it is important to underline that in the group randomized to ICD testing, 99 out of 1,218 patients had eventually an unsuccessful test, despite all the undertaken measures, which are unfortunately not discussed in great detail. The real value of PDT depends on its ability to identify patients with unsatisfactory safety margin of defibrillation energy, but it only makes sense if we react in a way that allows to achieve finally the efficacy as close to $100 \%$ as possible, that is, for example, placement of an additional subcutaneous lead or changing the device for a higher-voltage model.

At the same time, one should appreciate the fact that a successful PDT does not guarantee the $100 \%$ efficacy of the system in the future. The energy levels required to terminate VF may vary depending on the clinical situation. That fact might explain the occurrence of SCD in a certain percentage of ICD recipients $[16,17]$. Therefore, it is crucial to identify the group of patients, in whom a PDT is particularly needed, because of the high risk of system malfunction. Our data show that contemporarily "system malfunction" means in most cases the high DFT. We did not analyze our cohort in the search of risk factors for high DFT, but those were previously identified in other publications [11]. In conclusion, it may be reasonable to limit the PDT to the group of patients at risk of high DFT.

\section{Conclusions}

Contemporary technical solutions used in ICD systems, compared to previous years, allow to avoid most sensing disorders and ensure appropriate VF detection. The reason that justifies ICD defibrillation testing is the need to confirm the efficacy of high-voltage therapy. Despite increasingly high-energy values of the defibrillation impulse and available adjustments of its characteristics, there is still a group of patients who require additional procedures to achieve the appropriate defibrillation safety margin. That condition may be identified only by means of a pre-discharge test of the implanted ICD system.

\section{Conflict of interest: None declared}

\section{References}

1. Michowitz Y, Lellouche N, Contractor T et al. Defibrillation threshold testing fails to show clinical benefit during long-term follow-up of patients undergoing cardiac resynchronization therapy defibrillator implantation. Europace, 2011; 13 : 683-688.

2. Sauer WH, Lowery CM, Bargas RL et al. Utility of postoperative testing of implantable cardioverter-defibrillators. Pacing Clin Electrophysiol, 2011; 34: 186-192. 
3. Weiss DN, Zilo P, Luceri RM, Platt S, Rosenbaum M. Predischarge arrhythmia induction testing of implantable defibrillators may be unnecessary in selected cases. Am J Cardiol, 1997; 80: 1562-1565.

4. Glikson M, Luria D, Friedman PA et al. Are routine arrhythmia in ductions necessary in patients with pectoral implantable cardioverter defibrillators? J Cardiovasc Electrophysiol, 2000; 11: 127-135.

5. Viskin S, Rosso R. The top 10 reasons to avoid defibrillation threshold testing during ICD implantation. Heart Rhythm, 2008; 5: 391-393.

6. Brigniole M, Occhetta E, Bongiorni MG et al. Clinical evaluation of defibrillation testing in an unselected population of 2,120 consecutive patients undergoing first implantable cardioverterdefibrillator implant. J Am Coll Cardiol, 2012; 60: 981-987.

7. Stavrakis S, Patel NH, Reynolds DW. Defibrillation threshold testing does not predict clinical outcomes during long-term follow-up: A meta-analysis. PACE, 2013; 36: 1402-1408.

8. Pires LA, Johnson KM. Intraoperative testing of the implantable cardioverter-defibrillator: How much is enough? J Cardiovasc Electrophysiol, 2006; 17: 140-145.

9. Blatt JA, Poole JE, Johnson GW et al. No benefit from defibrillation threshold testing in the SCD-HeFT (Sudden Cardiac Death in Heart Failure Trial). J Am Coll Cardiol, 2008; 52: 551-556.

10. Guenther M, Rauwolf T, Bruggemann B et al. Pre-hospital discharge testing after implantable cardioverter defibrillator implantation: A measure of safety or out of date? A retrospective analysis of 975 patients. Europace, 2012; 14: 217-223.

11. Healey JS, Hohnloser SH, Glikson M et al.; Shockless IMPLant Evaluation (SIMPLE) Investigators. Cardioverter defibrillator implantation without induction of ventricular fibrillation: A single-blind, non-inferiority, randomised controlled trial (SIMPLE). Lancet, 2015; 385: 785-791.

12. Panotopoulos P, Krum D, Axtell K, Dhala A, Sra J, Akhtar M, Deshpande S. Ventricular fibrillation sensing and detection by implantable defibrillators: Is one better than the others? A prospective, comparative study. J Cardiovasc Electrophysiol, 2001; 12: 453-454.

13. Natale A, Sra J, Axtell K et al. Undetected ventricular fibrillation in transvenous implantable cardioverter-defibrillators. Prospective comparison of different lead system-device combinations. Circulation, 1996; 93: 91-98.

14. Osswald BR, De Simone R, Most S, Tochtermann U, Tanzeem A, Karck M. High defibrillation threshold in patients with implantable defibrillator: How effective is the subcutaneous finger lead? Eur J Cardiothorac Surg, 2009; 35: 489-492.

15. Verma A, Kaplan AJ, Sarak B et al. Incidence of very high defibrillation thresholds (DFT) and efficacy of subcutaneous (SQ) array insertion during implantable cardioverter defibrillator (ICD) implantation. J Interv Card Electrophysiol, 2010; 29: 127-133.

16. Greenberg H, Case RB, Moss AJ, Brown MW, Carroll ER, Andrews ML; MADIT-II Investigators. Analysis of mortality events in the Multicenter Automatic Defibrillator Implantation Trial (MADIT-II). J Am Coll Cardiol, 2004; 21: 1459-1465.

17. Carson P, Anand I, O'Connor C et al. Mode of death in advanced heart failure: The Comparison of Medical, Pacing, and Defibrillation Therapies in Heart Failure (COMPANION) trial. J Am Coll Cardiol, 2005; 46: 2329-2334. 